\title{
The introduction of simple cardiorespiratory fitness testing in overweight/obese type 2 diabetics: a pilot study
}

\author{
Lubica Cibičková ${ }^{1}$, David Karásek ${ }^{1}$, Jiří Lukeš ${ }^{2}$ and Norbert Cibiček ${ }^{3,4}$ \\ ${ }^{1}$ Department of Internal Medicine III - Nephrology, Rheumatology and Endocrinology, University Hospital, Olomouc, \\ Czech Republic \\ ${ }^{2}$ Department of Clinical Biochemistry, University Hospital, Olomouc, Czech Republic \\ ${ }^{3}$ Department of Medical Chemistry and Biochemistry, Faculty of Medicine and Dentistry, Palacky University, Olomouc, \\ Czech Republic \\ ${ }^{4}$ Department of Exercise Medicine and Cardiovascular Rehabilitation, University Hospital, Olomouc, Czech Republic
}

\begin{abstract}
Background: Low level of cardiorespiratory fitness has been recognized as an important independent and modifiable risk factor of increased morbidity and mortality. However, in standard outpatient settings, patients are not routinely screened for fitness and advantages of such testing for the management of type 2 diabetes have not been defined. Aim: To describe the toleration of a fast, simple and practicable fitness test (2-min step-inplace test) by overweight/obese type 2 diabetics and their performance indicated by 2-min step-in-place test score (STS). To study short-term anthropometric, functional and metabolic changes following the implementation of the test in the selected population. Methods: A total of 33 overweight/obese type 2 diabetics underwent, besides routine examination at the outpatient clinic, the fitness test (group A). Patients were asked to increase their regular physical activity with focus on walking without change in diet and chronic medication. Three to four months later, the subjects were tested again. An identical number of age- and sex-matched obese diabetics followed in our outpatient clinic (without fitness testing), was randomly selected from the Hospital Information System (control group B). Findings: All patients subjected to fitness testing completed the protocol successfully. STS score was found to have a considerable range with differences between males and females at the borderline of statistical significance. The data are compliant with lower aerobic endurance of obese diabetics compared with healthy population. Within study period, the tested group presented with improvements in STS (referring especially to the males) as well as in several laboratory parameters of glucose and lipid homeostasis, glomerular function and subclinical inflammation with no reflection in anthropometry. Group B demonstrated no significant change. In conclusion, 2-min step-in-place test is fast, undemanding and well-tolerated by patients and personnel. Following its validation based on cardiopulmonary exercise testing, the test may prove recommendable for screening or self-monitoring purposes.
\end{abstract}

Key words: fitness; insulin resistance; lipid metabolism; obesity; step-in-place test; type 2 diabetes

Received 10 January 2017; revised 19 August 2017; accepted 8 December 2017; first published online 14 January 2018

Correspondence to: Norbert Cibiček, Department of Medical Chemistry and Biochemistry, Faculty of Medicine and Dentistry, Hněvotínská 3, 77515 Olomouc, Czech Republic. Email: norbert.cibicek@upol.cz

\section{Introduction}

Low level of physical activity and cardiorespiratory fitness has recently been recognized and widely 
accepted as an independent modifiable risk factor of increased morbidity and premature cardiovascular and all-cause mortality (Blair et al.,1989; Lee et al., 1998; Kodama et al., 2009; Barry et al., 2014; McAuley and Beavers, 2014; Bouchard et al., 2015) in healthy (Blair et al., 1989), obese (Wei et al., 1999; Church et al., 2005) and/or diabetic patients (Wei et al., 2000). It is notable that the significance of the contribution of cardiorespiratory fitness to morbidity and mortality prediction exceeds that of 'classical' cardiovascular disease risk factors such as smoking, cholesterol, hypertension, obesity or diabetes (Church et al., 2005; Després, 2016). Therefore, the level of individual fitness was recommended for incorporation into cardiovascular disease risk factor evaluation and management (Israel et al., 2016; Ross et al., 2016). In spite of impressive body of evidence corroborating the need for large-scale fitness testing, patients' aerobic endurance is not routinely screened as advantages of such testing for the management of type 2 diabetes have not been clearly defined.

Current approach to the screening and identification of inactive individuals with poor fitness level in outpatient clinics may involve the use of an appropriate standardized physical activity questionnaire. Nonetheless, self-report questionnaires, albeit fast, inexpensive and convenient, are not employed routinely, perhaps due to bias with subjective perception leading to possible physical activity overestimation (Prince et al., 2008; Garriguet and Colley, 2014; Brett et al., 2015) or for being less strongly associated with cardio-metabolic risk variables and risk prediction scores than cardiorespiratory fitness assessed using an exercise test (Gray et al., 2015). Objective measures of physical activity include the use of an accelerometer. Using this tool, however, physical activity is usually monitored for seven consecutive days (Brett et al., 2015; Olsson et al., 2015), making the examination long and impractical for routine screening purposes. Direct evaluation of cardiorespiratory fitness is based on cardiopulmonary exercise testing or spiroergometry (Herdy et al., 2016). Owing to technical reasons such as sophisticated equipment (with expensive gas analyser) requiring trained personnel, the univocal indications for this 'golden standard' are limited.

With increasing frequency of obesity and type 2 diabetes in paediatric as well as adult population of the developed world (Cruz et al., 2005; Stevens et al., 2012) it becomes imperative to effectively stratify high-risk (ie, obese, diabetic and unfit) individuals by introducing fitness screening tests. These tests have to be economical, convenient for everyday outpatient routine and should be widely applicable for most patients, especially for obese diabetics. If such testing is established, the awareness of an integer reflecting the subject's level of fitness may, presumably, act also psychologically as an aid to put additional emphasis on regular physical activity and encourage the patients for increased involvement in self-management, thus reinforcing the current pharmacological treatment of the disease (Kokkinos et al., 2013). To address the aforementioned issues, a number of simple tests of functional fitness or aerobic endurance have been developed and standardized, for example 6-min walk test (Różańska-Kirschke et al., 2006) or Chester Step Test (Sykes and Roberts, 2004).

The present pilot study was designed with the intention to introduce a 2-min step-in-place test into the routine outpatient management of overweight/obese type 2 diabetics in stabilized condition. This test was opted for due to its fast and simple protocol practicable for temporally and spatially restricted outpatient settings (Jones and Rikli, 2002; Różańska-Kirschke et al., 2006). As the test was originally proposed for seniors and it has not been validated for the population in question thus far, we aimed at describing the toleration of the test by our overweight/obese type 2 diabetics and their performance indicated by 2-min step-in-place test score (STS). We were also interested to know if the introduction of the new mode of examination could be accompanied by any beneficial effect on anthropometric [eg, body mass index (BMI)], functional (resting systolic blood pressure and STS) or laboratory parameters (eg, blood glucose) in the short term. Therefore, the measurements were taken two times in three- to four-month period and the results were compared with randomly selected age- and sex-matched obese diabetic controls followed at our workplace in a standard regimen without fitness testing.

\section{Methods}

\section{Study population}

During the year 2016, in total 66 overweight or obese diabetic patients (50 males and 16 females, aged 36-77 and 30-66, respectively) followed up at 
the outpatient's surgery of the Department of Internal Medicine III - Nephrology, Rheumatology and Endocrinology, University Hospital, Olomouc, were enrolled in the study. The selection of the patients was based on fulfilling the inclusion criteria that were as follows: age between 30 and 80 years, presence of type 2 diabetes mellitus and overweight/obesity (ie, BMI $>25 \mathrm{~kg} / \mathrm{m}^{2}$ and/or waist circumference $>102 \mathrm{~cm}$ for males or $>88 \mathrm{~cm}$ for females). The diagnosis of diabetes had to be established for at least three months with no documented indication of coronary heart disease. The patients had to be clinically stabilized on standard antidiabetic therapy with no concomitant use of $\beta$-blockers. For group $\mathrm{A}$, the criteria also included the willingness of the subjects to participate in the study declared by signing the informed consent. Due to markedly lower frequency of female entries the enrolment (applicable for group A only) has finished as soon as the accepted statistical minimum of eight women were recruited. The exclusion criteria for group A comprised the incidence of any event or condition requiring hospitalization during the three to four-month follow-up, acute change in chronic medication (owing to deteriorated compensation of diabetes, hypertensive crisis, severe infection, etc.) and indispositions decreasing the validity of the endurance test or participant's ability/readiness to undergo it (eg, musculoskeletal trauma associated with pain perception, escalated psychological stress or loss of interest). Volunteers in group A (25 males and eight females aged 36-77 and 30-66, respectively, see Table 1) underwent, besides standard procedures, fitness testing. Group B was composed of an identical number of age- and sex-matched peers followed at our outpatient clinic without fitness testing. The group was formed post hoc by a professional blinded to the results of group A. Its database was created with the aid of hospital information system by random selection of patients fulfilling the aforementioned criteria with the exception of the time taken between two consecutive visits (for technical reasons this was increased to three to six months). No informed consent was required from group $\mathrm{B}$.

\section{Study design}

Standard services provided by the outpatient's surgery clinic (applicable for group B) included the following. First, harvesting of overnight fasting blood (sometimes accompanied also by the first morning urine) samples for routine laboratory markers of diabetes compensation and kidney function (for details refer to Tab., group B). Second, taking patients' history - concisely, focused on subjective complaints and possible pharmacological or lifestyle changes in the last several months. Third, simple physical examination involving the measurement of resting blood pressure and basic anthropometric parameters used to calculate BMI (body weight in kilograms/height in meters ${ }^{2}$ ). Finally, complex evaluation of the data and the patient's condition followed by necessary lifestyle recommendations (usually involving provision of educative brochures) and prescription of medication. Under normal circumstances the next visit took place three to four (up to six) months later.

The study protocol for group A encompassed additional examinations and laboratory tests. Markers of insulin resistance and cardiovascular risk factors included, besides routine parameters, also fasting plasma insulin, C-peptide, apolipoprotein B, high-sensitivity C-reactive protein and urinary albumin-creatinine ratio (for detailed list of parameters refer to Tab., group A). Waist and hip circumference were measured to calculate waist-to-hip ratio (WHR). Lifestyle was enquired in a more detailed manner, however, no standardized questionnaire was used. Following 2-min step-inplace test, the patients were individually educated about the relevance of cardiorespiratory fitness (as well as about the consequences of the opposite) and encouraged to increase their regular physical activity with focus on walking. The instructor's approach was strictly personalized, depending on the motivation or ability of the individual to cooperate in the long-term by introducing a small, yet realistic and tolerable change (typically an addition of two 30-min walks per week) in lifestyle without urgent modification of dietary habits (ie, collaborative goal setting, Peyrot and Rubin, 2007). The next visit took place three to four months later.

\section{Fitness testing protocol}

2-min step-in-place test was performed as described previously (Jones and Rikli, 2002; RóżańskaKirschke et al., 2006). Briefly, patients were calmed down for at least $5 \mathrm{~min}$ in a sitting position. 
Table 1 Characteristics of groups A and B with between-group and within-group comparisons

\begin{tabular}{|c|c|c|c|c|c|c|c|c|}
\hline \multirow[t]{2}{*}{ Parameter (unit) } & \multicolumn{2}{|c|}{$\begin{array}{l}\text { Entry data }(X) \\
(\text { mean } \pm \text { SD) }\end{array}$} & \multicolumn{2}{|l|}{$\begin{array}{l}\text { Final data }(\mathrm{Y}) \\
(\text { mean } \pm \mathrm{SD})\end{array}$} & \multicolumn{2}{|c|}{$\begin{array}{l}\text { Data difference }(\mathrm{Y}-\mathrm{X}) \\
\text { ( } n=\text { number of measurements) }\end{array}$} & \multicolumn{2}{|c|}{$\begin{array}{l}P \text {-value for trend } \\
\text { (within-group } \\
\text { comparisons) }\end{array}$} \\
\hline & Group A & Group B & Group A & Group B & Group A & Group B & Group A & Group B \\
\hline $\begin{array}{l}\text { Age (years, whole group) } \\
\text { Males }(n=25) \\
\text { Females }(n=8)\end{array}$ & $\begin{array}{l}51 \pm 11 \\
52 \pm 11 \\
51 \pm 11\end{array}$ & $\begin{array}{l}51 \pm 11 \\
52 \pm 11 \\
50 \pm 12\end{array}$ & $\begin{array}{l}51 \pm 11 \\
52 \pm 11 \\
51 \pm 11\end{array}$ & $\begin{array}{l}51 \pm 11 \\
52 \pm 11 \\
50 \pm 12\end{array}$ & $\begin{array}{l}- \\
- \\
-\end{array}$ & $\begin{array}{l}- \\
- \\
-\end{array}$ & $\begin{array}{l}- \\
- \\
-\end{array}$ & $\begin{array}{l}- \\
- \\
-\end{array}$ \\
\hline \multicolumn{9}{|l|}{ Anthropometry } \\
\hline Body weight $(\mathrm{kg})$ & $104 \pm 21$ & $104 \pm 24$ & $103 \pm 22$ & $104 \pm 24^{n}$ & $-0.59(n=33)$ & $+0.12(n=33)^{n}$ & 0.505 & $0.765^{\mathrm{a}}$ \\
\hline Body mass index $\left(\mathrm{kg} / \mathrm{m}^{2}\right)$ & $33.9 \pm 6.38$ & $32.1 \pm 6.11^{\mathrm{n}}$ & $33.7 \pm 6.6$ & $32.1 \pm 6.17$ & $-0.16(n=33)$ & $+0.06(n=33)^{n}$ & 0.563 & $0.638^{\mathrm{a}}$ \\
\hline Waist circumference $(\mathrm{cm})$ & $115 \pm 16.7$ & - & $114 \pm 17.2$ & - & $-1.58(n=33)$ & - & 0.102 & - \\
\hline Waist-to-hip ratio & $1.02 \pm 0.08$ & - & $1.01 \pm 0.08$ & - & $-0.01(n=33)$ & - & 0.139 & - \\
\hline \multicolumn{8}{|l|}{ Biochemistry } & $>0.999^{a}$ \\
\hline Glycated haemoglobin $\left(\mathrm{HbA}_{1 \mathrm{c}}, \mathrm{mmol} / \mathrm{mol}\right)$ & $59 \pm 26^{n}$ & $53 \pm 14$ & $47 \pm 15^{n}$ & $53 \pm 15$ & $-12(n=30)^{\mathrm{n}}$ & $-0.5(n=32)^{\mathrm{n} * * *}$ & $<0.0001^{\mathrm{a}}$ & 0.822 \\
\hline $\mathrm{FPG}(\mathrm{mmol} / \mathrm{L})$ & $8.9 \pm 3.7^{n}$ & $8.1 \pm 2.6^{n}$ & $7.5 \pm 3.0^{n}$ & $8.8 \pm 3.7$ & $-1.3(n=33)^{n}$ & $+0.8(n=29)^{n *}$ & $0.020^{\mathrm{a}}$ & $0.393^{\mathrm{a}}$ \\
\hline Insulin (mlU/L) & $38+45^{n}$ & - & $28 \pm 25^{n}$ & - & $-9.7(n=26)^{n}$ & - & $0.380^{\mathrm{a}}$ & - \\
\hline C-peptide (pmol/L) & $1197 \pm 839^{n}$ & - & $1003 \pm 597$ & - & $-194(n=29)^{n}$ & - & $0.143^{\mathrm{a}}$ & - \\
\hline HOMA-IR index (mmol $\left.\mathrm{mlU} / \mathrm{L}^{2}\right)$ & $18+25^{n}$ & - & $11+15^{n}$ & - & $-6.9(n=26)^{n}$ & - & $0.237^{\mathrm{a}}$ & - \\
\hline $\mathrm{TC}(\mathrm{mmol} / \mathrm{L})$ & $4.8 \pm 1.7^{n}$ & $4.5 \pm 1.0$ & $4.5 \pm 1.2$ & $4.5 \pm 1.1$ & $-0.3(n=33)^{n}$ & $0.0(n=33)$ & $0.322^{\mathrm{a}}$ & 0.914 \\
\hline LDL cholesterol (mmol/L) & $2.6 \pm 0.7$ & $2.5 \pm 0.7$ & $2.4 \pm 0.7$ & $2.4 \pm 0.8$ & $-0.2(n=30)^{n}$ & $-0.1(n=31)$ & 0.128 & 0.266 \\
\hline Apo B $(g / L)$ & $0.97 \pm 0.21$ & - & $0.88 \pm 0.20$ & - & $-0.09(n=25)^{n}$ & - & 0.017 & - \\
\hline TG (mmol/L) & $2.9+5.0^{\mathrm{n}}$ & $1.9 \pm 0.9$ & $2.1 \pm 2.0^{n}$ & $2.2 \pm 1.3$ & $-0.8(n=33)^{n}$ & $+0.3(n=33)^{n *}$ & $0.175^{\mathrm{a}}$ & 0.191 \\
\hline AIP & $0.25+0.43$ & $0.18+0.26$ & $0.14+0.37$ & $0.21+0.33$ & $-0.10(n=33)$ & $+0.03(n=33)^{*}$ & 0.025 & 0.434 \\
\hline HDL-cholesterol (mmol/L) & $1.09 \pm 0.34$ & $1.16 \pm 0.30$ & $1.19 \pm 0.35$ & $1.15 \pm 0.29$ & $+0.10(n=33)$ & $0.00(n=33)^{*}$ & 0.005 & 0.875 \\
\hline Non-HDL-cholesterol (mmol/L) & $3.7 \pm 1.7^{n}$ & $3.3 \pm 0.9$ & $3.3 \pm 1.2^{n}$ & $3.3 \pm 1.0$ & $-0.4(n=33)^{n}$ & $0.0(n=33)$ & $0.102^{a}$ & 0.892 \\
\hline hsCRP (g/L) & $4.7+4.9^{n}$ & - & $3.0 \pm 2.9^{n}$ & - & $-1.7(n=33)^{n}$ & - & $0.013^{a}$ & - \\
\hline Creatinine $(\mu \mathrm{mol} / \mathrm{L})$ & $83.2 \pm 19.9$ & $80.1 \pm 17.7$ & $82 \pm 20.3^{n}$ & $81.8 \pm 18.4$ & $-1.21(n=33)$ & $+1.75(n=32)$ & $0.276^{\mathrm{a}}$ & 0.075 \\
\hline eGFR $\left(\mathrm{ml} / \mathrm{s} / 1.73 \mathrm{~m}^{2}\right)$ & $1.2 \pm 0.3$ & $1.2 \pm 0.1$ & $1.3 \pm 0.3^{n}$ & $1.2 \pm 0.2$ & $+0.07(n=14)$ & $-0.01(n=14)^{*}$ & $0.021^{\mathrm{a}}$ & 0.630 \\
\hline $\mathrm{ACR}(\mathrm{mg} / \mathrm{mmol})$ & $13+41^{n}$ & $3.7 \pm 2.9$ & $6.2+16^{n}$ & $3.2 \pm 2.5$ & $-6.6(n=25)^{n}$ & $-0.5(n=6)$ & $0.025^{\mathrm{a}}$ & 0.605 \\
\hline \multicolumn{9}{|l|}{ Aerobic endurance (fitness) } \\
\hline 2-min STS & $84 \pm 16$ & - & $93 \pm 19$ & - & $+9(n=33)$ & - & $<0.0001$ & - \\
\hline
\end{tabular}

All data are presented, for clarity, as mean \pm SD (index ${ }^{n}$ indicates that Kolmogorov-Smirnov normality test was not passed). $P$-values for within-group comparisons are given. Student's $t$-test or Wilcoxon matched-pairs signed-ranks test (where indicated by index ${ }^{a}$ ) was utilized. Differences between respective changes in group A versus changes in group B were tested using unpaired $t$-test or Mann-Whitney Test (where indicated by index ${ }^{b}$. Symbols * and $* *$ denote $P<0.05$ and $P<0.001$, respectively. Some variables in group B (eg, hsCRP) were considered unnecessary for routine follow-up of the patients. Hence, these data were missing in the Hospital Information System. (The analyses in group A were made possible due to grant support.) Lower number of measurements were largely due to the character of the calculation employed (eGFR $>1.5 \mathrm{ml} / \mathrm{s} / 1.73 \mathrm{~m}{ }^{2}$ ). In case of other parameters (especially ACR, Apo B and insulin) the reasons were mainly technical (such as analytical interference).

$\mathrm{FPG}=$ fasting plasma glucose; $\mathrm{TC}=$ total cholesterol; $\mathrm{Apo} \mathrm{B}=\mathrm{Apolipoprotein} \mathrm{B} ; \mathrm{TG}=$ triglycerides; $\mathrm{AIP}=\mathrm{atherogenic}$ index of plasma; hsCRP = high-sensitivity C-reactive protein; eGFR = estimated GFR; $A C R=$ albumin-creatinine ratio; STS = step-in-place test score. 
Following instruction, the subjects stood up and started stepping on a place, alternately raising both knees, with maximum tolerated frequency, to a point midway between the patella and the iliac crest. The patients could stabilize themselves by holding tight to a chair, a piece of furniture or leaning against the wall. 2-min step-in-place test score (STS) was defined as the number of times right knee reached the required height within $2 \mathrm{~min}$.

\section{Clinical chemistry and calculations}

Biochemical analyses of glycated haemoglobin $\left(\mathrm{HbA}_{1 \mathrm{c}}\right)$, fasting plasma glucose (FPG), human insulin, C-peptide, total cholesterol (TC), triglycerides (TG), high density lipoprotein-cholesterol (HDL), apolipoprotein B (Apo B), high-sensitivity C-reactive protein (hsCRP), albumin and creatinine were performed at the Department of Clinical Biochemistry, University Hospital, Olomouc in accordance with standard protocols subjected to valid directives. No sample required a freezingthawing cycle.

Index of insulin resistance - homeostasis model assessment (HOMA-IR) was calculated as glucose $\times$ insulin/22.5 (Matthews et al., 1985). Non-HDL was calculated as TC-HDL. Low-density lipoprotein cholesterol (LDL) was calculated, where applicable (TG of $<4.5 \mathrm{mmol} / \mathrm{L}$ ), using Friedewald formula (Friedewald et al., 1972). Atherogenic index of plasma (AIP) was calculated as log (TG/HDL) (Frohlich and Dobiasova, 2003). Estimated glomerular filtration rate (eGFR) was calculated, where applicable, using the 2009 Chronic Kidney Disease Epidemiology Collaboration (CKD-EPI) creatinine equation with plasma creatinine, age, ethnicity and sex (Levey et al., 2009).

\section{Statistical analyses}

Statistical analyses were performed using GraphPad InStat software version 3.06 (GraphPad Software, San Diego, CA, USA). Data distribution was tested for normality by Kolmogorov-Smirnov test. Unless otherwise noted, data are expressed as means \pm standard deviation (SD). For paired data (ie, within-group comparisons) Student's $t$-test or, where appropriate, (nonparametric) Wilcoxon matched-pairs signed-ranks test were utilized. For comparisons between males and females or between-group comparisons of changes recorded over time in group A versus group B, unpaired $t$-test or (nonparametric) Mann-Whitney Test were applied. In each case, two-tailed $P$-value was selected. Selected level of significance was $P<0.05$.

\section{Results}

Regarding group A, all patients completed the fitness test successfully with no clinical complication. Differences between absolute values of STS in males versus females $(85 \pm 16$ in males versus $82 \pm 19$ in females at entry, and $96 \pm 19$ in males versus $86 \pm 18$ in females three to four months later) were statistically insignificant, although significant increment $(P<0.001)$ could be observed in males in contrast to females $(P=0.13)$. There were five males and two females aged above 60 in our cohort. Compared with heathy seniors of adequate age (Jones and Rikli, 2002; Różańska-Kirschke et al., 2006), the performance of the seniors in our cohort was as follows. At entry, two males and one female were below average and three males and one female were at average. Three to four months later, one male and one female remained below average, two males and one female remained at average, one male advanced to average and one male progressed above average. At entry, 10 (out of 20) males and two (out of six) females of $<60$ years of age did not reach average fitness level of healthy 60-64-yearold seniors. In total, group A presented with a significant increment in STS $(P<0.0001)$. The results are summarized (in combination with other parameters) in Table 1.

Group A also presented with significantly improved markers of glucose and lipid homeostasis (decreased FPG and $\mathrm{HbA}_{1 \mathrm{c}}$, lowered Apo B and AIP and elevated HDL), mitigated inflammation (hsCRP) and augmented glomerular function (rise in eGFR with fall in ACR). In addition, these changes were accompanied by statistically insignificant trends for reduction in a number of other parameters, such as waist circumference, TG, non-HDL or C-peptide. Decreases in WHR and BMI were unsubstantial. There were no statistically significant trends documented in Group B. When compared with those noted in group A, however, the directions had deleterious clinical connotations: $\mathrm{HbA}_{1 \mathrm{c}}$ decreased significantly less than in group A, 
FPG, TG and AIP increased producing significant differences from the changes distinguished in group A. The changes in HDL and eGFR were also significantly lower than those recognized in group A. In general, the data indicate positive development in group A, whereas group B remained globally unchanged (Table 1).

\section{Discussion}

Poor cardiorespiratory fitness is an independent risk factor for cardiovascular morbidity and cardiac or all-cause mortality. Long-term weight loss, decreased blood pressure and lipids as well as enhanced cardiorespiratory fitness are regarded as the most powerful steps to prevent macrovascular diabetic complications in type 2 diabetics (Boulé et al., 2003; Tanasescu et al., 2003). Therefore, regular physical activity is a fundamental part of the complex therapeutic approach to diabetics with influence on insulin resistance (Svačinová, 2007). In order to assess the level of physical activity, estimate individual fitness and predict the associated mortality risk in daily praxis, either standardized questionnaires or exercise testing can be used. In our study, we have decided to apply the simple 2-min step-in-place test without a standardized questionnaire. This decision was motivated by reports showing that poor fitness, rather than reported physical inactivity, is independently associated with increased mortality risk (Lee et al., 2011). Other data suggest that the level of physical activity determined by the General Practice Physical Activity Questionnaire tool reveals less associations with markers of cardiovascular disease or type 2 diabetes mellitus compared with cardiorespiratory fitness evaluated by a simple test (Gray et al., 2015). Therefore, it seems reasonable to propose that a simple physiological metric should be incorporated as a vital sign in cardiovascular disease risk factor evaluation and management (Després, 2016). For this purpose we have opted for a simple and convenient 2-min step-in-place test enabling us to put, as immediate feedback, additional emphasis on the relevance of being physically fit for life's quality and longevity.

As all participants comprehended and successfully completed the protocol with no documented clinical complication attributable to the test, we can see a few obstacles for its wide implementation within the scope of preventive diabetology. In agreement with previous findings (Hansen et al., 2012), STS indicated a somewhat lower level of cardiorespiratory fitness of obese type 2 diabetics compared with healthy subjects of comparable or higher age categories (Jones and Rikli, 2002; Różańska-Kirschke et al., 2006). Contrary to healthy seniors (Jones and Rikli, 2002; Różańska-Kirschke et al., 2006), no significant gender-dependent differences in the absolute values of STS were found as far as the whole cohort was concerned. This may be, at least in part, explained by the low number of female entries. As to females, a rather reluctant attitude towards being physically stressed was experienced. The reasons were mostly subjective and psychological (such as embarrassment).

One of the most interesting findings of this study was the statistically significant enhancement of the volunteers' performance over time. However, statistical significance was lost when females were considered separately. Another noteworthy observation was the favourable development in several biochemical markers confined to group A (Table 1). Although accompanied by (but not necessarily related to) increased aerobic endurance, the complex laboratory improvement was not reflected in anthropometric parameters. If augmented physical activity was the cause, lower FPG and $\mathrm{HbA}_{1 \mathrm{c}}$ might have resulted from more effective action of insulin at the adipocytes without concomitant reduction in body weight and fat stores (Hughes et al., 1993; Kelley and Goodpaster, 1999). The enhancement of insulin action is an important (and often overlooked) salutary effect suggesting that physical activity may be as efficacious in preventing insulin resistance as is losing body weight. Low level of cardiorespiratory fitness associated with physical inactivity is typical for patients with insulin resistance and results in higher risk of cardiovascular disease than obesity itself - a phenomenon that may shortly be put as 'fit and fat is better than unfit and unfat' (Lee et al., 1999). Concerning dyslipidaemia, the studied population presented with a typical diabetic profile involving high $\mathrm{TG}$ and low HDL with almost normal total and LDL cholesterol. Following the introduction of fitness testing, the levels of apoB and AIP decreased and the level of HDL rose in group A patients as opposed to their counterparts in group B (Table 1). It would be speculative to 
account for these differences using the limited data obtained. hsCRP, as a sensitive marker of subclinical inflammation, belongs among established predictors of cardiovascular risk associated with insulin resistance, metabolic syndrome, hyperglycaemia and type 2 diabetes (Rivero et al., 2009). In parallel to the elevation of STS, the level of subclinical inflammation subsided three to four months following the first fitness examination in group A. Even though physical activity and exercise training have been associated with reductions in hsCRP, we cannot ascertain as to how far these activities in the tested group had changed and, moreover, even if they did change, the mechanism whereby such interventions could lead to a reduction in hsCRP remains unclear (Hammonds et al., 2016). Exercise training was found to possess a potential to increase kidney function (Baria et al., 2014). Despite a lower number of measurements mainly due to eGFR exceeding $1.5 \mathrm{ml} / \mathrm{s} / 1.73 \mathrm{~m}^{2}$ the data (eGFR and ACR) suggest an improvement in glomerular function in the tested population. Although no significant difference was observed in group $\mathrm{B}$, it is notable that the trends in creatinine and eGFR were clinically unfavourable and in the case of the latter parameter, they were significantly different from the tested group A (Table 1).

Although a direct causal relationship may exist between aerobic endurance and several metabolic markers (Schwingshackl et al., 2013), the laboratory progress observed in group A can be, as for this study, exclusively attributed neither to physical fitness, nor to the introduction of fitness testing per se, for a number of reasons. Individuals in group A were all ready to undergo the fitness test - a fact that would probably not be true for all group B counterparts. Some evidence indicates that patient trust in physician and desire for involvement and collaborating care may be associated with better self-care behaviours, adherence and positive diabetes outcomes including biochemical markers such as FPG or $\mathrm{HbA}_{1 \mathrm{c}}$ (Lee and Lin, 2009; Charles, 2015). Furthermore, a lack of employment of a standardized cardiorespiratory fitness method opened a possibility of patient dissimulation, especially during the final examination. Based on marked discrepancies repeatedly found between subjective and objective measures of physical activity (questionnaires versus accelerometers, Brett et al., 2015), reported glycaemia (self-report diaries versus glucometer memory, Armstrong, 1991) and food intake (reported energy intake in dietary history questionnaires versus resting metabolic rate, Fricker et al., 1992), self-enhancement may be expected to be abundant in obese diabetics. Therefore, the reasons behind improvements in group A are likely to be multifactorial, involving, besides the introduction of fitness testing, a number of lifestyle factors (unmonitored in this study) and psychological influences such as self-motivation, willingness to compete or to please the researchers. Some of these elements (eg, competitiveness) may have played more important roles in males than in females. The investigation of the extent to which the respective factors might have participated on the favourable outcomes would require a different study design employing a multivariate analysis.

To be fit-for-purpose, 2-min step-in-place test requires better characterization and validation for obese diabetics using a widely accepted indicator of cardiorespiratory fitness, namely peak oxygen consumption, $\mathrm{VO}_{2 \text { peak }}$, as determined by cardiopulmonary exercise testing (Herdy et al., 2016). Subsequent effort should also address the question of objective measurement of physical activity (eg, using an accelerometer) and the need for study population enlargement.

\section{Conclusion}

2-min step-in-place test is temporally and spatially undemanding and well-tolerated by patients and personnel. STS score of overweight/obese type 2 diabetics was found to have a considerable range with differences between males and females at the borderline of statistical significance for the cohort studied. In general, the data are compliant with lower cardiorespiratory fitness of obese type 2 diabetics compared with healthy population. Within three to four-month period, the tested group presented with improvements in STS (referring especially to the males) as well as in several laboratory parameters of glucose and lipid homeostasis, glomerular function and subclinical inflammation with no reflection in anthropometry. Further effort is warranted to better characterize and validate the fitness test for obese diabetics so that it could become recommendable for future implementation into screening strategies or for self-monitoring purposes. Subsequent studies should also investigate the possibility of an 
existence of a causal relationship between the implementation of aerobic endurance testing into routine services provided by outpatient surgery clinics and the benefits observed.

\section{Acknowledgements}

The authors acknowledge skilful technical assistance of our pre-graduate students (E.R. and D.H.) during data processing and valuable correspondence with doctor M.B. in the phase of manuscript preparation.

\section{Financial Support}

This study was supported by grants $\mathrm{MH} \mathrm{CZ} \mathrm{-}$ DRO (FNOL, 00098892) - 87-62 from the Czech Ministry of Health and IGA-LF-2016-014 from the Palacky University, Olomouc, Czech Republic.

\section{Conflicts of Interest}

None.

\section{Ethical Standards}

The authors assert that all procedures contributing to this work comply with the ethical standards of the relevant national and institutional guidelines on human experimentation and with the Helsinki Declaration of 1975, as revised in 2008. The study was approved by the Local Ethics Committee of the University Hospital, Olomouc, Czech Republic (Ref. No.: 147/16). Before inclusion into the study, all active participants provided their informed consent.

\section{References}

Armstrong, D. 1991: The social context of technology in diabetes care: "compliance" and "control". Introduction. In Bradley, C., Christie, M. and Home, P., editors. Technology of diabetes care: converging medical and psychosocial perspectives. Chur, Switzerland: Harwood Academic Publishers, 17-24.

Baria, F., Kamimura, M.A., Aoike, D.T., Ammirati, A., Rocha, M.L., De Mello, M.T. and Cuppari, L. 2014: Randomized controlled trial to evaluate the impact of aerobic exercise on visceral fat in overweight chronic kidney disease patients. Nephrology, Dialysis, Transplantation 29, 857-64.

Barry, V.W., Baruth, M., Beets, M.W., Durstine, J.L., Liu, J. and Blair, S.N. 2014: Fitness vs. fatness on all-cause mortality: a meta-analysis. Progress in Cardiovascular Diseases 56, 382-90.

Blair, S.N., Kohl, H.W., Paffenbarger, R.S. Jr., Clark, D.G., Cooper, K.H. and Gobbons, L.W. 1989: Physical fitness and allcause mortality: a prospective study of healthy men and women. Journal of the American Medical Association 262, 2395-401.

Bouchard, C., Blair, S.N. and Katzmarzyk, P.T. 2015: Less sitting, more physical activity, or higher fitness? Mayo Clinic Proceedings 90, 1533-40.

Boulé, N.G., Kenny, G.P., Haddad, D., Wells, G.A. and Sigal, R.J. 2003: Meta-analysis of the effect of structured exercise training on cardiorespiratory fitness in type 2 diabetes mellitus. Diabetologia 46, 1071-81.

Brett, K.E., Wilson, S., Ferraro, Z.M. and Adamo, K.B. 2015: Self-report pregnancy physical activity questionnaire overestimates physical activity. Canadian Journal of Public Health 106, e297-302.

Charles, S. 2015: Implications of the patient-physician relationship for people with diabetes, thesis. Dietrich College of Humanities and Social Sciences.

Church, T.S., LaMonte, M.J., Barlow, C.E. and Blair, S.N. 2005: Cardiorespiratory fitness and body mass index as predictors of cardiovascular disease mortality among men with diabetes. Archives of Internal Medicine 165, 2114-20.

Cruz, M.L., Shaibi, G.Q., Weigensberg, M.J., Spruijt-Metz, D., Ball, G.D. and Goran, M.I. 2005: Pediatric obesity and insulin resistance: chronic disease risk and implications for treatment and prevention beyond body weight modification. Annual Review of Nutrition 25, 435-68.

Després, J.P. 2016: Physical activity, sedentary behaviours, and cardiovascular health: when will cardiorespiratory fitness become a vital sign? Canadian Journal of Cardiology 32, 505-13.

Fricker, J., Baelde, D., Igoin-Apfelbaum, L., Huet, J.M. and Apfelbaum, M. 1992: Underreporting of food intake in obese "small eaters". Appetite 19, 273-83.

Friedewald, W.T., Levy, R.I. and Fredrickson, D.S. 1972: Estimation of the concentration of low-density lipoprotein cholesterol in plasma, without use of the preparative ultracentrifuge. Clinical Chemistry 18, 499-502.

Frohlich, J. and Dobiasova, M. 2003: Fractional esterification rate of cholesterol and ratio of triglycerides to HDLcholesterol are powerful predictors of positive findings on coronary angiography. Clinical Chemistry 49, 1873-80.

Garriguet, D. and Colley, R.C. 2014: A comparison of selfreported leisure-time physical activity and measured moderate-to-vigorous physical activity in adolescents and adults. Health Reports 25, 3-11.

Gray, B.J., Stephens, J.W., Williams, S.P., Davies, C.A., Turner, D. and Bracken, R.M., Prosiect Sir Gâr Group 2015: Cardiorespiratory fitness is a stronger indicator of cardiometabolic risk factors and risk prediction than self-reported physical activity levels. Diabetes and Vascular Disease Research 12, 428-35.

Hammonds, T.L., Gathright, E.C., Goldstein, C.M., Penn, M.S. and Hughes, J.W. 2016: Effects of exercise on c-reactive 
protein in healthy patients and in patients with heart disease: a meta-analysis. Heart and Lung: the Journal of Critical Care 45, 273-82.

Hansen, E., Landstad, B.J., Gundersen, K.T. and Svebak, S. 2012: The relative importance of aerobic capacity, physical activity and body mass index in impaired glucose tolerance and Type 2 diabetes. Vulnerable Groups \& Inclusion 3, http:// dx.doi.org/10.3402/vgi.v3i0.10232.

Herdy, A.H., Ritt, L.E., Stein, R., Araújo, C.G., Milani, M., Meneghelo, R.S., Ferraz, A.S., Hossri, C., Almeida, A.E., Fernandes-Silva, M.M. and Serra, S.M. 2016: Cardiopulmonary exercise test: background, applicability and interpretation. Arquivos Brasileiros de Cardiologia 107, 467-81.

Hughes, V.A., Fiatrone, M.A., Fielding, R.A., Kahn, B.B., Ferrara, C.M., Shepherd, P., Fisher, E.C., Wolfe, R.R., Elahi, D. and Evans, W.J. 1993: Exercise increases muscle GLUT-4 levels and insulin action in subjects with impaired glucose tolerance. American Journal of Physiology 264, E855-62.

Israel, A., Kivity, S., Sidi, Y., Segev, S., Berkovitch, A., Klempfner, R., Lavi, B., Goldenberg, I. and Maor, E. 2016: Use of exercise capacity to improve SCORE risk prediction model in asymptomatic adults. European Heart Journal 37, 2300-306.

Jones, C.J. and Rikli, R.E. 2002: Measuring functional fitness of older adults. The Journal on Active Aging 1, 24-30.

Kelley, D.E. and Goodpaster, B.H. 1999: Effects of physical activity on insulin action and glucose tolerance in obesity. Medicine and Science in Sports and Exercise 31, 619-23.

Kodama, S., Saito, K., Tanaka, S., Maki, M., Yachi, Y., Asumi, M., Sugawara, A., Totsuka, K., Shimano, H., Ohashi, Y., Yamada, N. and Sone, H. 2009: Cardiorespiratory fitness as a quantitative predictor of all-cause mortality and cardiovascular events in healthy men and women: a meta-analysis. Journal of the American Medical Association 301, 2024-35.

Kokkinos, P.F., Faselis, C., Myers, J., Panagiotakos, D. and Doumas, M. 2013: Interactive effects of fitness and statin treatment on mortality risk in veterans with dyslipidemia: a cohort study. Lancet 381, 394-99.

Lee, C.D., Jackson, A.S. and Blair, S.N. 1998: US weight guidelines: is it also important to consider cardiorespiratory fitness? International Journal of Obesity and Related Metabolic Disorders 22, S2-7.

Lee, D.C., Sui, X., Ortega, F.B., Kim, Y.S., Church, T.S., Winett, R.A., Ekelund, U., Katzmarzyk, P.T. and Blair, S.N. 2011: Comparisons of leisure-time physical activity and cardiorespiratory fitness as predictors of all-cause mortality in men and women. British Journal of Sports Medicine 45, 504-10.

Lee, D.D., Blair, S.N. and Jackson, A.S. 1999: Cardiorespiratory fitness, body composition, and all cause cardiovascular disease mortality in men. American Journal of Clinical Nutrition 69, 373-80.

Lee, Y.Y. and Lin, J.L. 2009: The effects of trust in physician on self-efficacy, adherence and diabetes outcomes. Social Science \& Medicine (1982) 68, 1060-68.
Levey, A.S., Stevens, L.A., Schmid, C.H., Zhang, Y.L., Castro, A.F. 3rd, Feldman, H.I., Kusek, J.W., Eggers, P., Van Lente, F., Greene, T. and Coresh, J., CKD-EPI (Chronic Kidney Disease Epidemiology Collaboration) 2009: A new equation to estimate glomerular filtration rate. Annals of Internal Medicine 150, 604-12.

Matthews, D.R., Hosker, J.P., Rudenski, A.S., Naylor, B.A., Treacher, D.F. and Turner, R.C. 1985: Homeostasis model assessment: insulin resistance and beta-cell function from fasting plasma glucose and insulin concentrations in man. Diabetologia 28, 412-19.

McAuley, P.A. and Beavers, K.M. 2014: Contribution of cardiorespiratory fitness to the obesity paradox. Progress in Cardiovascular Diseases 56, 434-40.

Olsson, S.J., Borjesson, M., Ekblom-Bak, E., Hemmingsson, E., Hellenius, M.L. and Kallings, L.V. 2015: Effects of the Swedish physical activity on prescription model on health related quality of life in overweight older adults: a randomized controlled trial. BioMed Central Public Health 15, 687-96.

Peyrot, M. and Rubin, R.R. 2007: Behavioral and psychosocial interventions in diabetes: a conceptual review. Diabetes Care 30, 2433-440.

Prince, S.A., Adamo, K.B., Hamel, M.E., Hardt, J., Connor Gorber, S. and Tremblay, M. 2008: A comparison of direct versus self-report measures for assessing physical activity in adults: a systematic review. The International Journal of Behavioral Nutrition and Physical Activity 5, 56-79.

Rivero, A., Mora, C., Muros, M., Garcia, J., Herrera, H. and Navarro-González, J.F. 2009: Pathogenic perspectives for the role of inflammation in diabetic nephropathy. Clinical Science (London) 116, 479-92.

Ross, R., Blair, S.N., Arena, R., Church, T.S., Després, J.P., Franklin, B.A., Haskell, W.L., Kaminsky, L.A., Levine, B.D., Lavie, C.J., Myers, J., Niebauer, J., Sallis, R., Sawada, S.S., Sui, X. and Wisløff, U. 2016: Importance of assessing cardiorespiratory fitness in clinical practice: a case for fitness as a clinical vital sign: a scientific statement from the American Heart Association. Circulation 134, e653-699.

Różańska-Kirschke, A., Kocur, P., Wilk, M. and Dylewicz, P. 2006: The Fullerton Fitness Test as an index of fitness in the elderly. Medical Rehabilitation 10, 9-16.

Schwingshackl, L., Dias, S., Strasser, B. and Hoffmann, G. 2013: Impact of different training modalities on anthropometric and metabolic characteristics in overweight/obese subjects: a systematic review and network meta-analysis. Public Library of Science one 8, e82853.

Stevens, G.A., Singh, G.M., Lu, Y., Dannaei, G., Lin, J.K., Finucane, M.M., Bahalim, A.N., McIntire, R.K., Gutierrez, H.R., Cowan, M., Paciorek, C.J., Farzadfar, F., Riley, L. and Ezzati, M., Global Burden of Metabolic Risk Factors of Chronic Diseases Collaborating Group (Body Mass Index) 2012: National, regional, and global trends in adult overweight and obesity prevalences. Population Health Metrics $10,22-37$. 
Svačinová, H. 2007: Exercise therapy for patients with metabolic syndrome. Vnitrní lékařství 53, 540-44.

Sykes, K. and Roberts, A. 2004: The Chester step test - a simple yet effective tool for the prediction of aerobic capacity. Physiotherapy 90, 183-88.

Tanasescu, M., Leitzmann, M.F., Rimm, E.G. and Hu, F.B. 2003: Physical activity in relation to cardiovascular disease and total mortality among men with type 2 diabetes. Circulation 107, 2435-39.
Wei, M., Gibbons, L.W., Kampert, J.B., Nichman, M.Z. and Blair, S.N. 2000: Low cardiorespiratory fitness and physical inactivity as predictors of mortality in men with type 2 diabetes. Annals of Internal Medicine 132, 605-11.

Wei, M., Kampert, J.B., Barlow, C.E., Nichaman, M.Z., Gibbons, L.W., Paffenbarger, R.S. Jr. and Blair, S.N. 1999: Relationship between low cardiorespiratory fitness and mortality in normal-weight, overweight, and obese men. Journal of the American Medical Association 282, 1547-53. 